\title{
Targeted treatment for chronic lymphocytic leukemia: clinical potential of obinutuzumab
}

This article was published in the following Dove Press journal:

Pharmacogenomics and Personalized Medicine

22 December 2014

Number of times this article has been viewed

\section{Lukáš Smolej}

4th Department of Internal Medicine - Hematology, University Hospital Hradec Králové and Charles University in Prague, Faculty of Medicine in Hradec Králové, Hradec Králové, Czech Republic
Correspondence: Lukáš Smolej 4th Department of Internal Medicine Hematology, University Hospital and Charles University in Prague, Faculty of Medicine in Hradec Králové, Sokolská 58I, 50005 Hradec Králové, Czech Republic

Tel +420495832649, +420495834688

Fax +4204958320II

Email lukas.smolej@fnhk.cz

\begin{abstract}
Introduction of targeted agents revolutionized the treatment of chronic lymphocytic leukemia (CLL) in the past decade. Addition of chimeric monoclonal anti-CD20 antibody rituximab to chemotherapy significantly improved efficacy including overall survival (OS) in untreated fit patients; humanized anti-CD52 antibody alemtuzumab and fully human anti-CD20 antibody ofatumumab lead to improvement in refractory disease. Novel small molecule inhibitors such as ibrutinib and idelalisib demonstrated excellent activity and were very recently licensed in relapsed/refractory CLL. Obinutuzumab (GA101) is the newest monoclonal antibody approved for the treatment of CLL. This novel, glycoengineered, type II humanized anti-CD20 antibody is characterized by enhanced antibody-dependent cellular cytotoxicity and direct induction of cell death compared to type I antibodies. Combination of obinutuzumab and chlorambucil yielded significantly better OS in comparison to chlorambucil monotherapy in untreated comorbid patients. These results led to approval of obinuzutumab for the treatment of CLL. Numerous clinical trials combining obinutuzumab with other cytotoxic drugs and novel small molecules are currently under way. This review focuses on the role of obinutuzumab in the treatment of CLL.
\end{abstract}

Keywords: chronic lymphocytic leukemia, anti-CD20 antibodies, chlorambucil, rituximab, ofatumumab, obinutuzumab, overall survival

\section{Introduction}

Chronic lymphocytic leukemia (CLL), the most frequent adult leukemia in Western population, ${ }^{1,2}$ is characterized by accumulation of mature-looking CD5+/19+/23+ B cells in bone marrow, peripheral blood, and lymphatic organs. ${ }^{3}$ CLL is predominantly a disease of the elderly population (median age at diagnosis 65-72 years), ${ }^{4,5}$ is considered incurable with currently available therapies, and its extraordinary feature is its extreme variability in clinical course with overall survival (OS) ranging from months to decades. ${ }^{6}$

\section{Targeted agents: revolution in the treatment of CLL}

We have witnessed revolutionary changes in the treatment of CLL during the past 10 years. Combination of fludarabine, cyclophosphamide, and anti-CD20 monoclonal antibody rituximab (FCR) demonstrated superiority over fludarabine and cyclophosphamide (FC), and therefore, became the standard treatment of physically fit CLL patients. ${ }^{7,8}$ Rituximab can also be successfully used in combination with other cytotoxic agents such as bendamustine or high-dose corticosteroids. ${ }^{9-11}$ Humanized anti-CD52 antibody alemtuzumab is indicated in fludarabine-refractory CLL ${ }^{12}$ and fully human anti-CD20 antibody ofatumumab in fludarabine- and alemtuzumab-refractory disease; ${ }^{13}$ 
also more recently in untreated frail patients. ${ }^{14}$ Allogeneic stem cell transplantation, the only potentially curative treatment option in CLL, has been used more frequently in fit patients with highly unfavorable clinical course, thanks to lower transplant-related mortality associated with reducedintensity conditioning. ${ }^{15}$ Novel orally available agents targeting the B-cell receptor signaling pathway, namely ibrutinib, a Bruton tyrosine kinase inhibitor and idelalisib, a phosphatidylinositol-3-kinase (PI3K) $\delta$ inhibitor, achieved remarkable results in pretreated CLL patients ${ }^{16-19}$ and have been recently approved for relapsed/refractory disease and first-line treatment of patients with TP53 mutation/deletion. Promising agents in development include venetoclax (ABT199, GDC-0199), an oral bcl-2 inhibitor; ${ }^{20}$ lenalidomide, an immunomodulatory agent targeting the tumor microenvironment; ${ }^{21}$ BTK inhibitors CC-292 22 and ONO-4059; ${ }^{23}$ and PI3K inhibitor IPI-145. ${ }^{24}$ Immunotherapy using genetically engineered autologous $\mathrm{T}$ cells expressing chimerig antigen receptors (CARs) achieved excellent results in refractory CLL, but severe toxicity is a serious issue. ${ }^{25}$ Obinutuzumab, the first glycoengineered type II anti-CD20 antibody, shows unique features in mechanism of action and represents the third generation of monoclonal antibodies. This review focuses on the role of obinutuzumab in CLL (Table 1).

\section{Characteristics, mechanism of action, and in vitro activity of obinutuzumab}

Obinutuzumab (GA101, RO5072759, RG-7159, formerly afutuzumab) is a humanized, type II glycoengineered monoclonal anti-CD20 IgG1 antibody with calculated molecular weight of $146 \mathrm{kDa} .^{26}$ It was developed from a parental murine IgG1-kappa Bly1 antibody. ${ }^{27}$ Modifications included humanization and glycoengineering of the Fc region by producing the antibody in Chinese hamster ovary cells overexpressing the recombinant glycosylation enzymes $\beta 1-4-N$-acetylglucosaminyltransferase III and Golgi alfa-mannosidase II, resulting in the presence of complex, non-fucosylated oligosaccharides attached to Fc region. ${ }^{28}$

Monoclonal antibodies in general have three possible mechanisms of action. These include: 1) antibody-dependent cellular cytotoxicity (ADCC), 2) complement-dependent cytotoxicity (CDC), and 3 ) direct growth inhibition and apoptosis, referred to as direct cell death (DCD) (Figure 1). ${ }^{29}$ Type II characteristics of obinutuzumab, namely stronger ADCC and direct cell death induction as well as weaker CDC were repeatedly demonstrated in comparison to known type I antibodies rituximab and ofatumumab. Enhanced ADCC was achieved by modifying the Fc fragment of obinutuzumab by glycoengineering (modification of glycosylation leading to afucosylated Fc fragment) and by amino acid substitution, which led to augmented binding to both high- and low-affinity FC $\gamma$ RIII on effector cells (eg, NK-cells and macrophages), resulting in ADCC up to 100 -fold higher than rituximab. ${ }^{30,31}$ Interestingly, antibodydependent cellular phagocytosis (ADCP) by macrophages was weaker with obinutuzumab than that with rituximab and ofatumumab. ${ }^{32}$ Obinutuzumab does not localize to membrane lipid microdomains on the target cells; in addition, it binds markedly less $\mathrm{C} 1 \mathrm{q}$ than rituximab. ${ }^{33}$ The consequence is decreased CDC when compared to rituximab, especially ofatumumab. ${ }^{28}$ Immunofluorescence studies on Ramos cells showed that while rituximab was present at sites corresponding to lipid rafts, obinutuzumab formed stable complexes with CD20 at sites of cell-to-cell contact; this could be the explanation of stronger homotypic aggregation of target cells by obinutuzumab - another type II antibody feature. ${ }^{34}$ Different binding of type I vs type II antibodies also explain why B-cells can accommodate twice the number of type I antibodies compared with type II at saturating concentrations. ${ }^{28}$ Binding experiments showed that obinutuzumab recognizes a distinct but overlapping epitope of CD20 antigen compared

Table I Targeted agents for the treatment of CLL

\begin{tabular}{lll}
\hline Agent & Mechanism of action & Phase of development \\
\hline Obinutuzumab (GAI0I) & Glycoengineered type II anti-CD20 antibody \\
Ibrutinib (PCI-32765) & Bruton tyrosine kinase inhibition \\
CC-292 & Bruton tyrosine kinase inhibition \\
ONO-4059 & Bruton tyrosine kinase inhibition & Approved; Phase III \\
Idelalisib (GS-II0I) & Phosphatidylinositol-3-kinase $\delta$ inhibition \\
IPI-I45 & Phosphatidylinositol-3-kinase $\delta$ inhibition \\
Lenalidomide & Complex- microenvironment \\
Venetoclax (ABT-I99, GDC-0I99) & Bcl-2 inhibition \\
CAR T-cells & T cells with chimeric anti CDI9 receptor \\
\hline
\end{tabular}

Abbreviation: CLL, chronic lymphocytic leukemia. 


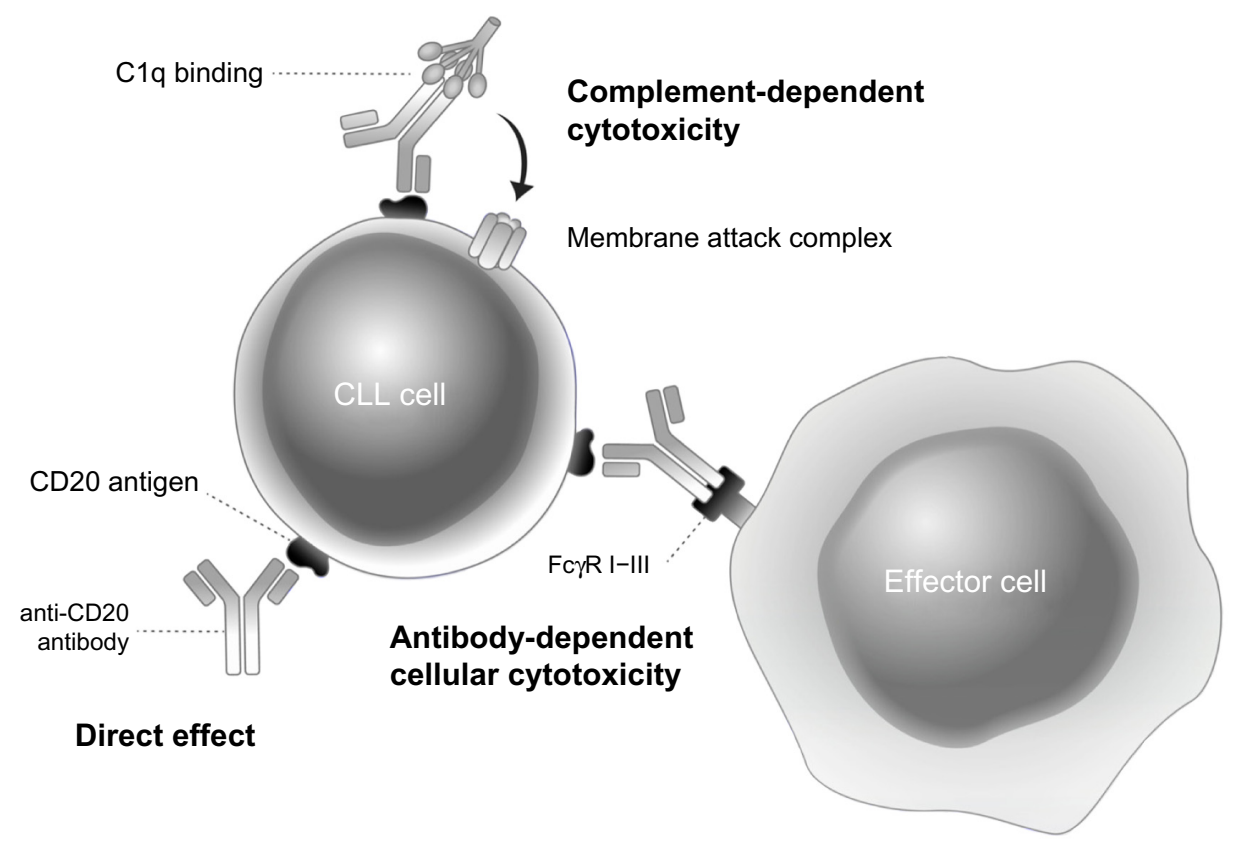

Figure I Mechanisms of action of anti-CD20 monoclonal antibodies.

Abbreviation: CLL, chronic lymphocytic leukemia.

to rituximab. ${ }^{28,34}$ Nevertheless, obinutuzumab binds in a different space orientation and with a wider elbow-hinge angle (by $30^{\circ}$ ) than rituximab. ${ }^{34}$ This is a consequence of amino acid sequence modification (substitution of valin for leucin at Kabat position 11) in immunoglobulin heavy chain elbow-hinge region - the site that affects the flexibility of the $\mathrm{Fab}^{\prime}$ and $\mathrm{F}(\mathrm{ab})^{\prime} 2$ antibody domains. Reversal of this change resulted in partial loss of the type II antibody attributes, emphasizing the importance of spatial structure of the elbow-hinge region for the function of type II antibodies. ${ }^{28}$ Obinutuzumab is a potent direct cell death inductor as demonstrated by ability to kill CLL cells without the presence of a cross-linking antibody. ${ }^{32}$ Proposed mechanisms of DCD include caspase-independent apoptosis ${ }^{28}$ and nonapoptotic, actin-dependent, lysosome-mediated cell death involving reactive oxygen species. ${ }^{35,36}$ As a result of enhanced ADCC and DCD, obinutuzumab achieved superior B-cell depletion to rituximab in healthy volunteers and whole blood assays from CLL patients (Table 2). ${ }^{28}$

\section{Pharmacodynamics}

In preclinical animal studies, obinutuzumab yielded strong anti-tumor effects in human lymphoma (diffuse large B-cell, mantle cell, and follicular lymphoma) xenograft models in SCID mice..$^{28,30,37}$ Intravenous obinutuzumab at the dose of $10 \mathrm{mg} / \mathrm{kg}$ and $30 \mathrm{mg} / \mathrm{kg}$ on days 1 and 8 effectively depleted B-cells from peripheral blood and was more effective than rituximab in B-cell depletion from spleen and lymph nodes in cynomolgus monkeys. ${ }^{28}$ Potent B-cell depletion by obinutuzumab was confirmed in whole blood assays from healthy volunteers and CLL or indolent lymphoma patients. ${ }^{28,38} \mathrm{In}$ a Phase I/II GAUGUIN study, all Phase I patients $(\mathrm{n}=13)$ with relapsed/refractory CLL achieved very rapid reduction in total B-cell count (normalization on day 8) after the first infusion of obinutuzumab; 12/13 patients achieved B-cell depletion. B-cell counts recovered in most of the patients within 24 months of the last obinutuzumab. ${ }^{39}$

Table 2 Comparison of commercially available anti-CD20 antibodies

\begin{tabular}{llll}
\hline Antibody & Obinutuzumab & Rituximab & Ofatumumab \\
\hline Trade name (EU) & Gazyvaro & MabThera & Arzerra \\
Manufacturer & Roche & Roche & GlaxoSmithKline \\
Antibody type & II & I & I \\
lgG subclass & $\operatorname{lgGl}$ & $\operatorname{lgGl}$ & $\operatorname{lgG} \mid$ \\
Structure & Humanized & Chimeric & Fully human \\
Binding to & Large loop & Large loop & Large and small \\
CD20 epitope & & & loop \\
Binding to & - & ++ & ++++ \\
lipid rafts & & & \\
ADCC & ++++ & ++ & ++ \\
CDC & + & ++ & ++++ \\
Direct cell death & ++++ & + & + \\
induction & & &
\end{tabular}

Abbreviations: $A D C C$, antibody-dependent cellular cytotoxicity; $C D C$, complement-dependent cytotoxicity; I lg, immunoglobulin. 


\section{Pharmacokinetics}

Pharmacokinetic (PK) data were investigated in relapsed/ refractory CLL patients treated within the GAUGUIN study. ${ }^{39}$ Mean obinutuzumab $\mathrm{C}_{\max }$ and $\mathrm{C}_{\text {trough }}$ concentrations raised with increasing dose (400-1,200 mg/dose). Steady state was achieved at cycle 2 by patients who received $1,000 \mathrm{mg}$ infusions on days 1,8 , and 15 at cycle 1 followed by 1,000 mg every 3 weeks. ${ }^{39}$ Notably, patients who achieved therapeutic response had higher obinutuzumab plasma concentrations compared to nonresponding patients; in addition, patients who failed to respond had higher $\mathrm{C}_{\max }$ to $\mathrm{C}_{\text {trough }}$ ratios, suggesting more rapid obinutuzumab clearance in these patients. ${ }^{40}$ The geometric mean distribution volume of obinutuzumab is approximately $3.8 \mathrm{~L}$, suggesting mainly intravascular distribution. PK parameters were not significantly influenced by age or decreased renal function. There are no data on PK in patients with severe renal insufficiency (creatinine clearance $<30 \mathrm{~mL} / \mathrm{min}$ ) or impaired liver function. Metabolic studies were not conducted with obinutuzumab.

\section{Efficacy}

\section{Phase I studies}

Two Phase I studies reported efficacy of obinutuzumab in relapsed/refractory CLL. Patients treated within the GAUGUIN Phase I/II study ${ }^{39}$ were administered obinutuzumab 400-1,200 $\mathrm{mg}$ iv at days 1 and 8 of cycle 1 and then repeated at day 1 of cycles $2-8$ for every 4 weeks ( $n=13$, Phase I) or received a fixed dose of $1,000 \mathrm{mg}$ iv at days 1,8 , and 15 of cycle 1 and repeated at day 1 of cycles 2-8 for every 3 weeks $(n=20$, Phase II). Median age of the patients was 64 years and 62.5 years. The patients were heavily pretreated with a median of three previous therapies including fludarabine in $91 \%$; bulky disease was present in $31 \%$ and $35 \%$ but advanced clinical stage (Binet C) was relatively infrequent $(8 \%$ and $11 \%$ ). Obinutuzumab achieved ORR of $62 \%$ (Phase I) and $15 \%$ (Phase II); there were no complete responses. Median progression-free survival (PFS) and response duration in Phase II were 10.7 months and 8.9 months, respectively. ${ }^{39}$ The GAUSS Phase I dose-escalation study enrolled 22 patients with relapsed/refractory lymphoproliferative diseases including seven patients with CLL/SLL. ${ }^{41}$ The regimen consisted of four weekly infusions of 100-1,200 mg (6 cohorts in 3+3 design) followed by obinutuzumab maintenance for every 3 months for a total of eleven doses in responding patients $(n=8)$. The ORR rate in CLL/SLL patients was $14 \%$ (PR in 1/7 patients).

\section{CLLII Phase III study}

The pivotal CLL11 Phase III study conducted by German CLL Study Group (GCLLSG) randomized 589 untreated patients with significant comorbidities (cumulative CIRS score $\geq 7 \mathrm{and} /$ or creatinine clearance $<70 \mathrm{~mL} / \mathrm{min}$ ) in 1:2:2 ratio between CLB monotherapy $(0.5 \mathrm{mg} / \mathrm{kg}$ at days 1 and 15 for a maximum of 6 cycles), CLB+rituximab (R-CLB, $375 \mathrm{mg} / \mathrm{m}^{2}$ iv at day 1 of cycle $1,500 \mathrm{mg} / \mathrm{m}^{2}$ at day 1 of cycles $\left.2-6\right)$, and CLB+obinutuzumab (G-CLB, $100 \mathrm{mg}$ iv at day $1,900 \mathrm{mg}$ iv at day $2,1,000 \mathrm{mg}$ iv at days 8 and 15 of cycle $1,1,000 \mathrm{mg}$ iv at day 1 of cycles 2-6). ${ }^{42}$ Patients who progressed during or within 6 months from the end of CLB monotherapy were allowed to crossover to G-CLB arm. Both combination regimens yielded significantly better results to CLB monotherapy in terms of CR rate (G-CLB, 22\%; R-CLB, 8\%; CLB, $0 \%$ ) and PFS (G-CLB, 23 months; R-CLB, 15.7 months; CLB, 10.9 months, $P<0.0001)$. Very importantly, patients treated with G-CLB had significantly longer OS in comparison to those treated with CLB alone (median OS was not reached in either group, death rate $9 \%$ vs $20 \%, P=0.002$ ). CR rate, minimal residual disease (MRD) negativity in peripheral blood measured by allele-specific oligonucleotide PCR, and PFS were also significantly improved with G-CLB vs R-CLB (CR 20.7\% vs 7.0\%, $P<0.001$; MRD negativity $37.7 \%$ vs $3.3 \%, P<0.001$; PFS median 26.7 months vs 15.2 months, $P<0.001$ ). However, it is important to note that the results of PFS and OS are not mature due to short median observation time of 18.7 months. Table 3 summarizes the patient characteristics, efficacy, and safety of

Table 3 Comparison of patient characteristics, efficacy, and toxicity of anti-CD20 antibody-chlorambucil combinations from CLLII study

\begin{tabular}{|c|c|c|c|}
\hline & R-CLB & G-CLB & $P$-value \\
\hline $\mathrm{n}$ & 233 & 238 & ns \\
\hline Median age, years & 73 & 74 & ns \\
\hline Males, \% & 64 & 59 & ns \\
\hline Binet stage C, \% & 36 & 36 & ns \\
\hline Median CIRS score & 8 & 8 & ns \\
\hline Median creatinine clearance, $\mathrm{mL} / \mathrm{min}$ & 61 & 61 & ns \\
\hline Unmutated IGHV, \% & 62 & 61 & ns \\
\hline FISH del I Iq/I7 p, \% & $19 / 5$ & $16 / 8$ & ns \\
\hline $\mathrm{B} 2 \mathrm{M}>3.5 \mathrm{mg} / \mathrm{L}, \%$ & 35 & 32 & ns \\
\hline Median cycles administered & 6 & 6 & ns \\
\hline ORR\% & 65 & 78 & $<0.001$ \\
\hline CR\% & 7 & 21 & $<0.001$ \\
\hline MRD negativity in peripheral blood, \% & 3 & 38 & $<0.001$ \\
\hline Median PFS, months & 15.2 & 26.7 & $<0.001$ \\
\hline Grade III/IV infusion-related reactions, \% & 4 & 20 & NR \\
\hline Grade III/IV neutropenia, \% & 28 & 33 & NR \\
\hline Grade III-V infections, \% & 14 & 12 & ns \\
\hline
\end{tabular}

Abbreviations: $O R R$, overall response rate; $C R$, complete response; $C L B$, chlorambucil; G, obinutuzumab; R, rituximab; B2M, $\beta 2$-microglobulin; IGHV, immunoglobulin heavy chain variable region; FISH, fluorescent in situ hybridization; MRD, minimal residual disease; ns, not significant $(P>0.01)$; NR, not reported; PFS, progression-free survival; CIRS, Cumulative Illness Rating Scale. 
R-CLB and G-CLB arms of the CLL11 trial. Obinutuzumab has been approved based on the results of the CLL11 trial for the first-line treatment of CLL in comorbid patients.

\section{Safety}

Infusion-related reactions (IRRs) were the predominant side effects in Phase I studies. In the GAUGUIN study, IRR rate was $96 \%$, with grade III infections in 30\% ${ }^{39}$ The most frequent IRR symptom was hypotension, followed by pyrexia, chills, and vomiting. Importantly, recommended premedication included only acetaminophen and antihistamine; corticosteroids were administered only for treatment of IRRs. Grade IV neutropenia occurred in 35\%, grade IV thrombocytopenia in 13\%, febrile neutropenia in $9 \%$, and grade III infections in $13 \%$ (no infections grade IV developed). There were no cases of tumor lysis syndrome. ${ }^{39}$ No dose-limiting toxicities were recorded in the GAUSS trial. IRR was the most frequent side effect (all grades, 73\%; grade III/IV, 18\%). ${ }^{41}$ In the CLL11 Phase III study safety run-in, 5/6 patients had grade I/II IRRs and the same number of patients developed grade III/IV neutropenia without fevers or infections. ${ }^{41}$ Final analysis revealed that obinuzutumab induced more frequent infusion-related reactions than rituximab (any grade, $66 \%$ vs $38 \%$; grade III/IV, $20 \%$ vs $4 \%$ ); nevertheless, grade III/IV IRRs occurred only during the first infusion and were not seen in subsequent cycles. Obinutuzumab also caused more frequent severe neutropenia than rituximab or CLB monotherapy ( $33 \%$ vs $28 \%$ vs $16 \%$ ); however, this did not result in increased rate of severe infections (12\% vs $14 \%$ vs $14 \%$ ). Grade III/IV thrombocytopenia was again most frequent with G-CLB (10\% vs 3\% vs $4 \%$ ) and grade III/IV anemia was rare ( $4 \%$ in all groups). ${ }^{43}$

\section{Recommendations for administration of obinutuzumab}

Obinutuzumab is supplied in 1,000 mg single-use vials at a concentration of $25 \mathrm{mg} / \mathrm{mL}$ as a sterile, colorless to slightly brown liquid concentrate without preservatives and with $\mathrm{pH}$ of 6.0. Obinutuzumab is administered as an intravenous infusion. The $1,000 \mathrm{mg}$ vial should be diluted in $250 \mathrm{~mL}$ of $0.9 \%$ sodium chloride solution (normal saline) under sterile conditions. The dosing schedule approved for CLL is $1,000 \mathrm{mg}$ flat dose on days 1,8 , and 15 of cycle 1 and on day 1 of cycles $2-6 .^{42}$ The infusions should be administered under the close supervision of an experienced physician and full resuscitation facilities should be immediately available. Prophylaxis of tumor lysis syndrome with adequate hydration and allopurinol should commence 12-24 hours before infusion, especially in patients with high tumor burden (eg, absolute lymphocyte count $\geq 25 \times 10^{9} / \mathrm{L}$ ). As IRRs are frequent with obinutuzumab and hypotension is the most frequent symptom, withholding antihypertensive treatment 12 hours before the infusion and during the infusion should be considered. ${ }^{42}$ Recommended premedication includes combination of oral analgesic/antipyretic, antihistaminic drug, and corticosteroid: eg, paracetamol 1,000 mg, diphenhydramine $50 \mathrm{mg}$, and methylprednisolone $80 \mathrm{mg} .{ }^{26}$ The first $1,000 \mathrm{mg}$ dose is divided into two parts. First, $100 \mathrm{mg}$ should be infused for 4 hours; remaining $900 \mathrm{mg}$ should be infused on the same day or on day 2 at an initial rate of $50 \mathrm{mg} / \mathrm{h}$ with further $50 \mathrm{mg}$ increments for every 30 minutes up to a maximal rate of $400 \mathrm{mg} / \mathrm{h}$. As IRRs did not correlate with absolute lymphocyte count or extent of lymphadenopathy in the CLL11 study, all patients irrespective of leukemic burden should be closely monitored for signs of IRR. ${ }^{42}$ In case of good tolerability, subsequent infusions can be started at the dose of $100 \mathrm{mg} / \mathrm{h}$ with $100 \mathrm{mg}$ increments for every 30 minutes up to the maximal rate of $400 \mathrm{mg} / \mathrm{h}$; this results in approximately 3 hours of infusion. ${ }^{42}$

\section{Conclusion}

Introduction of obinutuzumab represents a major step forward in the treatment of CLL patients. This novel type II antibody with unique properties characterized by enhanced ADCC and DCD proved its efficacy in combination with chlorambucil in comorbid untreated patients with CLL, and for the first time, achieved OS prolongation in this difficult-to-treat patient population. Therefore, obinutuzumab-chlorambucil should be considered as a standard regimen for first-line treatment of CLL patients unsuitable for full-dose FCR. The safety profile is acceptable but caution is needed particularly during first infusion, because IRRs are frequent and may be severe. Regular blood count monitoring for neutropenia is also necessary. We can expect that current clinical trials testing combination of obinutuzumab with other agents such as ibrutinib, idelalisib, venetoclax (GDC-0199), bendamustine, and others ${ }^{44}$ will broaden indications for CLL in near future.

\section{Acknowledgment}

Supported by grant IGA MH CZ NT13412-4/2012, by MH CZ - DRO (UHHK, 00179906) and by program PRVOUK P37/08. I thank Marie Smolej for graphic art design.

\section{Disclosure}

The author has received travel grants and/or honoraria from Roche, Janssen, Gilead, and GlaxoSmithKline. The author reports no other conflicts of interest in this work. 


\section{References}

1. Diehl LF, Karnell LH, Menck HR. The American College of Surgeons Commission on Cancer and the American Cancer Society. The national cancer data base report on age, gender, treatment, and outcomes of patients with chronic lymphocytic leukemia. Cancer. 1999;86(12): 2684-2692.

2. Nabhan C, Aschebrook-Kilfoy B, Chiu BC, et al. The impact of race, ethnicity, age and sex on clinical outcome in chronic lymphocytic leukemia: a comprehensive Surveillance, Epidemiology, and End Results analysis in the modern era. Leuk Lymphoma. 2014. Epub 2014 Apr 3.

3. Hallek M, Cheson BD, Catovsky D, et al; International Workshop on Chronic Lymphocytic Leukemia. Guidelines for the diagnosis and treatment of chronic lymphocytic leukemia: a report from the international workshop on chronic lymphocytic leukemia updating the National Cancer Institute-Working Group 1996 guidelines. Blood. 2008;111(12):5446-5456.

4. Dores GM, Anderson WF, Curtis RE, et al. Chronic lymphocytic leukaemia and small lymphocytic lymphoma: overview of the descriptive epidemiology. Br J Haematol. 2007;139(5):809-819.

5. Panovska A, Doubek M, Brychtova Y, Mayer J. Chronic lymphocytic leukemia and focusing on epidemiology and management in everyday hematologic practice: recent data from the Czech Leukemia Study Group for Life (CELL). Clin Lymphoma Myeloma Leuk. 2010;10(4): 297-300.

6. Rai KR, Sawitsky A, Cronkite EP, Chanana AD, Levy RN, Pasternack BS. Clinical staging of chronic lymphocytic leukemia. Blood. 1975;46(2):219-234.

7. Hallek M, Fischer K, Fingerle-Rowson G, et al; International Group of Investigators, German Chronic Lymphocytic Leukaemia Study Group. Addition of rituximab to fludarabine and cyclophosphamide in patients with chronic lymphocytic leukaemia: a randomised, open-label, phase 3 trial. Lancet. 2010;376(9747):1164-1174.

8. Robak T, Dmoszynska A, Solal-Céligny P, et al. Rituximab plus fludarabine and cyclophosphamide prolongs progression-free survival compared with fludarabine and cyclophosphamide alone in previously treated chronic lymphocytic leukemia. J Clin Oncol. 2010;28(10): 1756-1765.

9. Smolej L. The role of high-dose corticosteroids in the treatment of chronic lymphocytic leukemia. Expert Opin Investig Drugs. 2012;21(7): 1009-1017.

10. Fischer K, Cramer P, Busch R, et al. Bendamustine combined with rituximab in patients with relapsed and/or refractory chronic lymphocytic leukemia: a multicenter phase II trial of the German chronic lymphocytic leukemia study group. J Clin Oncol. 2011;29(26):3559-3566.

11. Fischer K, Cramer P, Busch R, et al. Bendamustine in combination with rituximab for previously untreated patients with chronic lymphocytic leukemia: a multicenter phase II trial of the German chronic lymphocytic leukemia study group. J Clin Oncol. 2012;30(26):3209-3216.

12. Keating MJ, Flinn I, Jain V, et al. Therapeutic role of alemtuzumab (Campath-1H) in patients who have failed fludarabine: results of a large international study. Blood. 2002;99(10):3554-3561.

13. Wierda WG, Kipps TJ, Mayer J, et al; Hx-CD20-406 Study Investigators. Ofatumumab as single-agent CD20 immunotherapy in fludarabine-refractory chronic lymphocytic leukemia. J Clin Oncol. 2010;28(10):1749-1755.

14. Hillmen P, Robak T, Janssens A, et al. Ofatumumab - chlorambucil versus chlorambucil alone in patients with untreated chronic lymphocytic leukemia (CLL): results of the phase III study complement-1 (OMB 110911). Blood. 2013;122:528.

15. Brown JR, Kim HT, Armand P, et al. Long-term follow-up of reduced-intensity allogeneic stem cell transplantation for chronic lymphocytic leukemia: prognostic model to predict outcome. Leukemia. 2013;27(2):362-369.

16. Byrd JC, Furman RR, Coutre SE, et al. Targeting BTK with ibrutinib in relapsed chronic lymphocytic leukemia. N Engl J Med. 2013;369(1): $32-42$.
17. Byrd JC, Brown JR, O'Brien S, et al; RESONATE Investigators. Ibrutinib versus ofatumumab in previously treated chronic lymphoid leukemia. N Engl J Med. 2014;371(3):213-223.

18. Brown JR, Byrd JC, Coutre SE, et al. Idelalisib, an inhibitor of phosphatidylinositol 3-kinase p110delta, for relapsed/refractory chronic lymphocytic leukemia. Blood. 2014;123(22):3390-3397.

19. Furman RR, Sharman JP, Coutre SE, et al. Idelalisib and rituximab in relapsed chronic lymphocytic leukemia. $N$ Engl J Med. 2014;370(11): 997-1007.

20. Vogler M, Dinsdale D, Dyer MJ, Cohen GM. ABT-199 selectively inhibits BCL2 but not BCL2L1 and efficiently induces apoptosis of chronic lymphocytic leukaemic cells but not platelets. Br J Haematol. 2013;163(1):139-142.

21. Kater AP, Tonino SH, Egle A, Ramsay AG. How does lenalidomide target the chronic lymphocytic leukemia microenvironment? Blood. 2014;124(14):2184-2189.

22. Evans EK, Tester R, Aslanian S, et al. Inhibition of Btk with CC-292 provides early pharmacodynamic assessment of activity in mice and humans. J Pharmacol Exp Ther. 2013;346(2):219-228.

23. Akinleye A, Chen Y, Mukhi N, Song Y, Liu D. Ibrutinib and novel BTK inhibitors in clinical development. J Hematol Oncol. 2013; 6:59.

24. Dong S, Guinn D, Dubovsky JA, et al. IPI-145 antagonizes intrinsic and extrinsic survival signals in chronic lymphocytic leukemia cells. Blood. 2014. Epub 2014 Sep 25.

25. Porter DL, Levine BL, Kalos M, Bagg A, June CH. Chimeric antigen receptor-modified T cells in chronic lymphoid leukemia. N Engl J Med. 2011;365(8):725-733.

26. Obinutuzumab (Gazyvaro). Summary of product characteristics. 2014. Available from: http://www.ema.europa.eu/docs/en_GB/ document_library/EPAR_-_Public_assessment_report/human/002799/ WC500171596.pdf. Accessed October 17, 2014.

27. Poppema S, Visser L. Preparation and application of monoclonal antibodies: B cell panel and parafin tissue reactive panel. Biotest Bull. 1987;3:131-139.

28. Mössner E, Brünker P, Moser S, et al. Increasing the efficacy of CD20 antibody therapy through the engineering of a new type II anti-CD20 antibody with enhanced direct and immune effector cell-mediated B-cell cytotoxicity. Blood. 2010;115(22):4393-4402.

29. Beers SA, Chan CH, French RR, Cragg MS, Glennie MJ. CD20 as a target for therapeutic type I and II monoclonal antibodies. Semin Hematol. 2010;47(2):107-114.

30. Herter S, Herting F, Mundigl O, et al. Preclinical activity of the type II CD20 antibody GA101 (obinutuzumab) compared with rituximab and ofatumumab in vitro and in xenograft models. Mol Cancer Ther. 2013;12(10):2031-2042.

31. Herting F, Friess T, Bader S, et al. Enhanced anti-tumor activity of the glycoengineered type II CD20 antibody obinutuzumab (GA101) in combination with chemotherapy in xenograft models of human lymphoma. Leuk Lymphoma. 2014;55(9):2151-5160.

32. Rafiq S, Butchar JP, Cheney C, et al. Comparative assessment of clinically utilized CD20-directed antibodies in chronic lymphocytic leukemia cells reveals divergent NK cell, monocyte, and macrophage properties. J Immunol. 2013;190(6):2702-2711.

33. Kern DJ, James BR, Blackwell S, Gassner C, Klein C, Weiner GJ. GA101 induces NK-cell activation and antibody-dependent cellular cytotoxicity more effectively than rituximab when complement is present. Leuk Lymphoma. 2013;54(11):2500-2505.

34. Niederfellner G, Lammens A, Mundigl O, et al. Epitope characterization and crystal structure of GA101 provide insights into the molecular basis for type I/II distinction of CD20 antibodies. Blood. 2011;118(2): 358-367.

35. Honeychurch J, Alduaij W, Azizyan M, et al. Antibody-induced nonapoptotic cell death in human lymphoma and leukemia cells is mediated through a novel reactive oxygen species-dependent pathway. Blood. 2012;119(15):3523-3533. 
36. Alduaij W, Ivanov A, Honeychurch J, et al. Novel type II anti-CD20 monoclonal antibody (GA101) evokes homotypic adhesion and actindependent, lysosome-mediated cell death in B-cell malignancies. Blood. 2011;117(17):4519-4529.

37. Dalle S, Reslan L, Besseyre de Horts T, et al. Preclinical studies on the mechanism of action and the anti-lymphoma activity of the novel anti-CD20 antibody GA101. Mol Cancer Ther. 2011;10(1):178-185.

38. Bologna L, Gotti E, Manganini M, et al. Mechanism of action of type II, glycoengineered, anti-CD20 monoclonal antibody GA101 in B-chronic lymphocytic leukemia whole blood assays in comparison with rituximab and alemtuzumab. J Immunol. 2011;186(6):3762-3769.

39. Cartron G, de Guibert S, Dilhuydy MS, et al. Obinutuzumab (GA101) in relapsed/refractory chronic lymphocytic leukemia: final data from the phase 1/2 GAUGUIN study. Blood. 2014;124(14):2196-2202.

40. Meneses-Lorente G, Carlile D, Birkett J, Wenger MK, Cartron G, Morschhauser F. Pharmacokinetics of RO5072759 (GA101) in patients with relapsed/refractory CD20+ malignant disease (phase I/II study BO20999). Blood. 2010;116:1833.
41. Sehn LH, Assouline SE, Stewart DA, et al. A phase 1 study of obinutuzumab induction followed by 2 years of maintenance in patients with relapsed CD20-positive B-cell malignancies. Blood. 2012;119(22): 5118-5125.

42. Goede V, Fischer K, Busch R, et al. Obinutuzumab plus chlorambucil in patients with CLL and coexisting conditions. $N$ Engl J Med. 2014;370(12):1101-1110.

43. Goede V, Fischer K, Busch R, et al. Chemoimmunotherapy with GA101 plus chlorambucil in patients with chronic lymphocytic leukemia and comorbidity: results of the CLL11 (BO21004) safety run-in. Leukemia. 2013;27(5):1172-1174.

44. US National Institutes of Health. 2014. Available from: http:// clinicaltrials.gov/ct2/results?term=obinutuzumab+AND+CLL\&recr= Open. Accessed October 22, 2014.
Pharmacogenomics and Personalized Medicine

\section{Publish your work in this journal}

Pharmacogenomics and Personalized Medicine is an international, peerreviewed, open access journal characterizing the influence of genotype on pharmacology leading to the development of personalized treatment programs and individualized drug selection for improved safety, efficacy and sustainability. This journal is indexed on the American Chemical

\section{Dovepress}

Society's Chemical Abstracts Service (CAS). The manuscript management system is completely online and includes a very quick and fair peer-review system, which is all easy to use. Visit http://www.dovepress. $\mathrm{com} /$ testimonials.php to read real quotes from published authors.

Submit your manuscript here: http://www.dovepress.com/pharmacogenomics-and-personalized-medicine-journal 